\title{
Fruit juice drinks prevent endogenous antioxidant response to high-fat meal ingestion
}

\author{
Cristiana Miglio ${ }^{1}$, Ilaria Peluso ${ }^{1}$, Anna Raguzzini ${ }^{1}$, Deborah V. Villaño ${ }^{2}$, Eleonora Cesqui ${ }^{1}$, \\ Giovina Catasta ${ }^{1}$, Elisabetta Toti $^{1}$ and Mauro Serafini ${ }^{1}$ * \\ ${ }^{1}$ Agricultural Research Council (CRA), Via Ardeatina 546, 00178 Rome, Italy \\ ${ }^{2}$ Food and Nutrition Unit, IRCCS San Raffaele Pisana, Via della Pisana 235, 00163 Rome, Italy
}

(Submitted 19 October 2012 - Final revision received 10 June 2013 - Accepted 23 June 2013 - First published online 12 August 2013)

\begin{abstract}
High-fat meals (HFM) induce metabolic stress, leading to the activation of protective mechanisms, including inflammation and endogenous antioxidant defences. In the present study, we investigated the effects of antioxidant-rich fruit juice drinks on the endogenous antioxidant response induced by HFM. In a double-blind, cross-over design ( $10 \mathrm{~d}$ washout), fourteen overweight volunteers were randomly assigned to one of the following interventions: HFM $+500 \mathrm{ml}$ placebo beverage (HFM-PB, free from fruit); HFM $+500 \mathrm{ml}$ antioxidant beverage 1 (HFM-AB1; apple, grape, blueberry and pomegranate juices and grape skin, grape seed and green tea extracts); HFM $+500 \mathrm{ml}$ antioxidant beverage 2 (HFM-AB2; pineapple, black currant and plum juices). HFM-PB consumption increased the plasma levels of thiols $(\mathrm{SH})(4 \mathrm{~h}, P<0 \cdot 001)$ and uric acid (UA) $(2 \mathrm{~h}, P<0 \cdot 01)$ and total radical-trapping antioxidant parameter (TRAP) $(4 \mathrm{~h}, P<0 \cdot 01)$. Following the consumption of drinks, UA production was significantly reduced with respect to placebo beverage consumption $8 \mathrm{~h}$ after $\mathrm{HFM}$-AB2 consumption $(P<0.05)$. SH levels were reduced $0.5(P<0.05), 1(P<0.05)$ and $2 \mathrm{~h}(P<0.01)$ after HFM-AB1 consumption and 2,4 and $8 \mathrm{~h}(P<0.05)$ after HFM-AB2 consumption. Plasma TRAP $(2 \mathrm{~h}, P<0 \cdot 001)$ and urinary ferric reducing antioxidant power $(0-8 \mathrm{~h}, P<0 \cdot 01)$ were increased by HFM-AB1 consumption, the drink with the highest in vitro antioxidant capacity, but not by HFM-AB2 consumption. In urine, UA levels were significantly increased from basal levels after the consumption of HFM-PB and HFM-AB2. However, neither of the beverages increased the urinary excretion of UA with respect to the placebo beverage. In conclusion, the increase in UA and SH levels induced by HFM as part of an endogenous antioxidant response to postprandial stress can be prevented by the concomitant ingestion of antioxidant-rich fruit juice drinks.
\end{abstract}

Key words: High-fat meals: Fruit juice drinks: Endogenous antioxidants: Non-enzymatic antioxidant capacity

The instauration of postprandial stress conditions following the ingestion of high-energy meals is recognised as one of the contributing factors to the development of $\mathrm{CVD}^{(1)}$. The underlying mechanism involves the excess production of pro-oxidant molecules, which, in turn, may inactivate endothelium-derived relaxing factors, in particular, NO, leading to impaired endothelium-dependent vasodilatation and to the onset of an inflammatory response, which further contributes to the generation of free radicals ${ }^{(2)}$. While high-carbohydrate meals are thought to increase inflammation in subjects with altered glucose and insulin metabolism ${ }^{(3)}$, postprandial hypertriacylglycerolaemia has been reported to be predictive for cardiovascular events and CHD incidence in healthy people ${ }^{(4)}$. In intervention studies, the ingestion of single high-fat meals (HFM) has been reported to be associated with inflammation ${ }^{(5-7)}$, impairment of endothelium-dependent vasodilatation $^{(8,9)}$ and increased oxidative stress $^{(9)}$ in both healthy and diabetic subjects.

Thus, excess fat intake can affect redox balance in healthy people, leading to an increased risk of vascular impairment. To the extent that antioxidants can provide protection from the harmful impact of pro-oxidant molecules ${ }^{(10)}$, understanding the redox mechanisms of defence activated by the body against postprandial stress could be of fundamental importance in the early prevention of CVD.

Humans can use a variety of protecting systems for both preventing and repairing oxidative damages, including enzymatic (superoxide dismutase and glutathione peroxidase) and non-enzymatic (e.g. dietary vitamin E, $\beta$-carotene and vitamin $\mathrm{C}$ and the endogenous antioxidants thiols $(\mathrm{SH})$ and uric acid (UA)) agents. While enzymes are mainly active in cells, the non-enzymatic antioxidant network comprises the

Abbreviations: AB1, antioxidant beverage 1; AB2, antioxidant beverage 2; FRAP, ferric reducing antioxidant power; HFM, high-fat meals; NEAC, non-enzymatic antioxidant capacity; PB, placebo beverage; SH, thiols; TRAP, total radical-trapping antioxidant parameter; UA, uric acid. 
most relevant antioxidant system in the plasma, with UA and protein thiol groups being the main contributors ${ }^{(11)}$. In a previous work, we had shown that the acute ingestion of a fat-rich meal designed to contain no antioxidants increased the levels of circulating TAG and total cholesterol as well as inflammatory cytokines, such as TNF- $\alpha$ and IL-6, leading to the onset of postprandial metabolic stress lasting for up to $8 \mathrm{~h}^{(6)}$. In the same work, for the first time, we had also shown that this meal-induced metabolic and inflammatory stress was accompanied by the activation of endogenous antioxidant mechanisms of defence provided by UA and $\mathrm{SH}$, without altering plasma vitamin and carotenoid concentrations ${ }^{(6)}$. It has recently been demonstrated that postprandial inflammation induced by high-fat and -sugar meals is attenuated by the concomitant ingestion of antioxidant-rich foods and beverages ${ }^{(1,7,12)}$. However, none of the studies has evaluated the effect of dietary antioxidants on the endogenous counterpart. In the present study, we aimed to investigate the effects of antioxidant-rich beverages on the endogenous antioxidant response induced by HFM in healthy overweight adults.

\section{Materials and methods}

\section{Materials}

The compounds 6-hydroxy-2,5,7,8-tetramethylchroman-2carboxylic acid (Trolox) and 2,4,6-tripyridyl-s-triazine (TPTZ) were purchased from Fluka. R-Phycoerythrin was purchased from Europa Bioproducts Limited; 2,2'-azobis(2-amidinopropane) dihydrochloride (ABAP) was purchased from Wako Chemical. PBS tablets, sodium acetate trihydrate, 5,5'-dithiobis(2-nitrobenzoic acid), glutathione and ethylenediaminetetraacetic acid were purchased from Sigma. Potassium dihydrogen phosphate was purchased from Carlo Erba; iron(III) chloride 6-hydrate and di-sodium hydrogen orthophosphate 12-hydrate were purchased from BDH. Ferrous sulphate 7-hydrate was purchased from Merck. All solvents used were of HPLC grade and purchased from Carlo Erba. High-purity water was obtained in the laboratory using an Alpha-Q system (Millipore).

\section{Methods}

Blood was collected in EDTA- and/or heparin-coated tubes and centrifuged immediately at $1300 \mathrm{~g}$ at $4^{\circ} \mathrm{C}$ for $15 \mathrm{~min}$, after which the plasma was separated and stored at $-80^{\circ} \mathrm{C}$. The total radical-trapping antioxidant parameter (TRAP) ${ }^{(13)}$ and the ferric reducing antioxidant power (FRAP) ${ }^{(14)}$ were used to measure the 'chain-breaking' and 'ferric reducing' antioxidant capacities, respectively, in both plasma and in vitro samples.

Determination of $\mathrm{SH}$ levels was performed using $5,5^{\prime}$-dithiobis(2-nitrobenzoic acid) ${ }^{(15)}$. Plasma UA levels were quantified enzymatically using colorimetric kits provided by Sentinel CH. SpA. Urinary creatinine levels were measured using Cayman Chemical Company colorimetric kits, according to the manufacturer's instructions; the kits were provided by Vinci-Biochem. The levels of 8 -iso-PG F2 $\alpha$ were assayed using as enzyme immunoassay kit (Assay Designs, provided by TEMA Ricerca).

The levels of vitamin $\mathrm{C}^{(16)}$, vitamin $\mathrm{E}$ and carotenoids in blood were measured by HPLC methods ${ }^{(17,18)}$. Flavonoids, measured by HPLC ${ }^{(19)}$, and total phenolics, measured by the Folin-Ciocalteu method ${ }^{(20)}$, used in the preparation of the two drinks were provided by 'The Coca Cola Company'.

\section{Experimental design}

Approval for the study was obtained from the Ethics Committee for Non-Clinical Research, and all procedures involving human subjects complied with the Declaration of Helsinki as revised in 2000 .

Inclusion criteria were healthy overweight adults, with a BMI ranging from 25 to $30 \mathrm{~kg} / \mathrm{m}^{2}$, not smoking and taking no supplements or drugs. Overweight subjects were selected in order to possibly minimise between-participant variability deriving from differences in body fat deposition and to increase the likelihood of an enhanced response to the high-fat meal. Written informed consent was obtained from all the participants.

The subjects were advised to follow a low-antioxidant diet, by avoiding fresh fruit and vegetables and their products, tea, coffee and wine, and to refrain from exercise $2 \mathrm{~d}$ before the study. Compliance to dietary instructions was evaluated through dietary questionnaires and interviews.

The study followed a cross-over, randomised design, with a $10 \mathrm{~d}$ washout period between treatments, in order to minimise possible carry-over biases. The subjects were randomly assigned to one of the following interventions: HFM $+500 \mathrm{ml}$ placebo beverage (HFM-PB); HFM $+500 \mathrm{ml}$ antioxidant beverage 1 (HFM-AB1); HFM $+500 \mathrm{ml}$ antioxidant beverage 2 (HFM-AB2). Following the washout period, interventions were repeated until all the subjects received all the three treatments in a random order.

On the day of the study, after an overnight fast, venous blood samples were collected before test meal and beverage ingestion $(0 \mathrm{~h})$ and at different time points $(0.5,1,2,4,6$ and $8 \mathrm{~h})$ from consumption. Urine samples were also collected before and 8,12 and $24 \mathrm{~h}$ after ingestion, in order to follow changes in the levels of selected biomarkers over a $24 \mathrm{~h}$ period.

\section{Test meal and fruit juice drink composition}

The macronutrient composition of the test meal is given in Table 1 . The energy of the meal, given as breakfast on three

Table 1. Test meal macronutrient composition and energy

\begin{tabular}{lccccccc}
\hline & Proteins $(\mathrm{g})$ & Lipids $(\mathrm{g})$ & Saturated lipids $(\mathrm{g})$ & Carbohydrates $(\mathrm{g})$ & Fibres $(\mathrm{g})$ & Energy $(\mathrm{kcal})$ & Energy $(\mathrm{kJ})$ \\
\hline Average & 52 & 81 & 37 & 104 & 3.1 & 1344 & 5623 \\
Energy $(\%)$ & 15 & 55 & & 29 & 1 & & \\
\hline
\end{tabular}


Table 2. Sugar content and antioxidant properties of the two fruit juice drinks

\begin{tabular}{|c|c|c|}
\hline & $\mathrm{AB} 1$ & AB2 \\
\hline \multicolumn{3}{|l|}{ Sugars $(g / l)$} \\
\hline Saccharose & 5.8 & 54 \\
\hline Glucose & 37 & 28 \\
\hline Fructose & 57 & 29 \\
\hline Sorbitol & $3 \cdot 2$ & 0.9 \\
\hline Total & 103 & 112 \\
\hline \multicolumn{3}{|l|}{ Phenolics (mg/l) } \\
\hline Anthocyanins & 13 & 32 \\
\hline Hydroxycinnamic acids & 45 & 0.5 \\
\hline Flavan-3-ols & 407 & 2.5 \\
\hline Flavonols & 49 & 20 \\
\hline Total phenolics* $(\mathrm{mM})$ & $10 \cdot 9$ & $5 \cdot 6$ \\
\hline TRAP† (mм) & $16 \cdot 4$ & 9.7 \\
\hline FRAP $\ddagger$ (mM) & $27 \cdot 6$ & $13 \cdot 6$ \\
\hline
\end{tabular}

$A B$, antioxidant beverage; TRAP, total radical-trapping antioxidant parameter; FRAP, ferric reducing antioxidant power.

* Gallic acid equivalents.

$\dagger \mathrm{mmol}$ Trolox equivalents.

$\ddagger \mathrm{mmol} \mathrm{Fe}^{2+}$ equivalents.

different days according to its co-ingestion with the placebo beverage or either of the two drinks, was $5623 \mathrm{~kJ}$ ( $1344 \mathrm{kcal}$ ) in total. According to the Italian Recommended Intakes of Nutrients and Energy (LARN) ${ }^{(21)}$, in a balanced diet, $55 \%$ of energy should derive from carbohydrates, $15 \%$ from proteins and $30 \%$ from fats. In the present study, in order to deliver a high-fat meal to the volunteers, we replaced carbohydrates with fats, resulting in a meal that contained $30 \%$ of energy from carbohydrates and $55 \%$ from fats. The ingestion of the same test meal has been shown to induce inflammatory reactions as well as endogenous antioxidant defences, mediated by UA and $\mathrm{SH}$, in a previous work by our group ${ }^{(6)}$.

In the selection of single food items, we attempted to avoid foods with a high purine content. During digestion, purines are metabolised into UA. Foods with a high purine content, such as meat and seafood, may cause a diet-induced increase in plasma UA levels after ingestion ${ }^{(22)}$, resulting in a possible overestimation of postprandial plasma non-enzymatic antioxidant capacity (NEAC) values. The test meal used in the present study comprised fried potatoes (212 g), fried eggs (108 g), cheese (90 g) and white bread $(90 \mathrm{~g})$, with a total purine content of $53 \mathrm{mg}^{(23)}$

In order to minimise changes in food composition, all food items were purchased at the same place and/or from the same company. In addition, between-day meal variability was reduced by following a detailed protocol for meal preparation, as described previously ${ }^{(6)}$.

The fruit juice drinks contained water, fruit juices, fruit extracts and sugars and no proteins, lipids or dietary fibres. The sugar and antioxidant composition of the drinks are reported in Table 2 . AB1 contained $86 \%$ of a mix of apple, grape, blueberry and pomegranate juices and grape skin, grape seed and green tea extracts. It contained high levels of flavan-3-ols $(407 \mathrm{mg} / \mathrm{l})$, along with smaller concentrations of hydroxycinnamic acids, flavonols and anthocyanins (Table 2). The total sugar content was $103 \mathrm{~g} / \mathrm{l}$, mainly fructose and glucose. We tested the effect of two distinct beverages, differing in their in vitro antioxidant capacities and total phenolic content, with the AB1 beverage showing the higher values. It contained $63 \%$ of a mix of pineapple, black currant and plum juices and mainly anthocyanins $(32 \mathrm{mg} / \mathrm{l})$. The total sugar content was $112 \mathrm{mg} / \mathrm{l}$, basically deriving from saccharose and fructose. The total phenolic content of AB1, as measured by the Folin-Ciocalteu assay, was twice that of AB2 (10.9 v. $5.6 \mathrm{~mm}$ ). Accordingly, AB1 displayed higher in vitro NEAC than $\mathrm{AB} 2$ (Table 2).

The placebo beverage contained water and a drink-matched quantity of sugars and was free from any fruit juice or fruit extracts. Accordingly, it did not display NEAC or have detectable levels of phenolic compounds.

\section{Statistical analysis}

Absolute changes (means with their standard errors) in plasma concentrations following meal intake were calculated as change $v$. baseline concentrations. Statistical analysis of the absolute changes was carried out using a two-way repeatedmeasures (two-factor repetition) ANOVA, with beverage and time as within-subject factors. Bonferroni post hoc analysis was used to determine differences between the treatments. This post hoc analysis was utilised to assess both mean differences among the three beverages within a single time point and intervention effects at different time points within the treatments with respect to baseline. All statistical evaluations were performed using the SigmaStat software (Jandel Scientific, Inc.).

\section{Results}

A total of fifteen adults aged $30-56$ years and with a mean BMI of $26 \cdot 8(\mathrm{SD} 2 \cdot 2) \mathrm{kg} / \mathrm{m}^{2}$ fulfilled the eligibility criteria and signed a written consent form to be included in the study. Among them, one subject withdrew from the study due

Table 3. Physical characteristics and baseline antioxidant biomarker profile of the subjects

(Mean values and standard deviations, $n$ 14)

\begin{tabular}{|c|c|c|}
\hline \multirow[b]{2}{*}{ Biomarkers } & \multicolumn{2}{|c|}{ Subjects } \\
\hline & Mean & SD \\
\hline BMI $\left(\mathrm{kg} / \mathrm{m}^{2}\right)$ & $26 \cdot 8$ & $2 \cdot 2$ \\
\hline Age (years) & 45 & 9 \\
\hline Glucose (mg/l) & 840 & 160 \\
\hline $\mathrm{TAG}(\mathrm{mg} / \mathrm{l})$ & 890 & 530 \\
\hline Total cholesterol (mg/l) & 1730 & 310 \\
\hline $\mathrm{UA}(\mu \mathrm{mol} / \mathrm{l})$ & 354 & 90 \\
\hline $\mathrm{SH}(\mu \mathrm{mol} / \mathrm{l})$ & 570 & 122 \\
\hline FRAP $(\mu \mathrm{mol} / \mathrm{l})$ & 1152 & 205 \\
\hline TRAP $(\mu \mathrm{mol} / \mathrm{l})$ & 1189 & 136 \\
\hline Vitamin C (mg/l) & $7 \cdot 1$ & 2.2 \\
\hline$\alpha$-Tocopherol (mg/l) & $11 \cdot 7$ & $3 \cdot 2$ \\
\hline Cryptoxanthin $(\mu \mathrm{g} / \mathrm{l})$ & 123 & 58 \\
\hline Lycopene $(\mu \mathrm{g} / \mathrm{l})$ & 570 & 160 \\
\hline$\alpha$-Carotene $(\mu \mathrm{g} / \mathrm{l})$ & 47 & 46 \\
\hline$\beta$-Carotene $(\mu \mathrm{g} / \mathrm{l})$ & 350 & 260 \\
\hline Urinary FRAP ( $\mu \mathrm{mol} / \mathrm{mg}$ creatinine) & $6 \cdot 0$ & 1.5 \\
\hline Urinary isoprostane (ng/mg creatinine) & $2 \cdot 6$ & 0.8 \\
\hline Urinary UA (mg/mg creatinine) & $0 \cdot 1$ & 0.09 \\
\hline
\end{tabular}

UA, uric acid; SH, thiols; FRAP, ferric reducing antioxidant power; TRAP, total radical-trapping antioxidant parameter. 

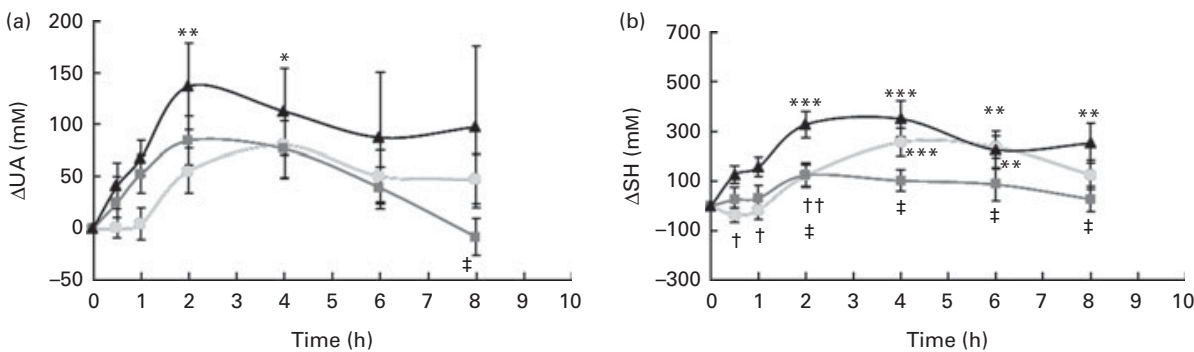

Fig. 1. Absolute changes ( $\Delta=$ change $v$. T0) in plasma (a) uric acid (UA) and (b) thiol (SH) levels following the consumption of the high-fat meal (HFM) + placebo beverage $(₫)$, HFM + antioxidant beverage 1 (-) and HFM + antioxidant beverage 2 (- - ) in fourteen healthy, overweight volunteers. Values are means, with their standard errors represented by vertical bars. Mean value was significantly different from that before meal intake: ${ }^{\star} P<0.05,{ }^{\star \star} P<0.01,{ }^{\star \star \star}{ }^{*} P<0.001$. Mean value for HFM + antioxidant beverage 1 was significantly different from that of the placebo beverage within a single time point: $\dagger P<0.05$, $\dagger \dagger P<0.01$. $\ddagger$ Mean value for HFM + antioxidant beverage 2 was significantly different from that of the placebo beverage within a single time point $(P<0.05)$.

to personal reasons and was excluded from the statistical analysis. Previous interventions with a similar group size of ten to fifteen subjects have been demonstrated to be adequately powered to detect statistically significant physiological impacts of either HFM alone or HFM and concomitant antioxidant beverage ingestion ${ }^{(6,7,24)}$.

The baseline characteristics of the subjects are reported in Table 3. When compared with the standard reference values (glucose 600-1100 mg/l, TAG $<1800 \mathrm{mg} / \mathrm{l}$ and total cholesterol $<2000 \mathrm{mg} / \mathrm{l}$ ), mean glucose, TAG and total cholesterol levels were found to be in normal ranges. Fasting plasma TRAP and FRAP ranges were 992-1409 and 747-1481 $\mu \mathrm{mol} / \mathrm{l}$, respectively. Mean plasma ascorbic acid, $\alpha$-tocopherol and UA levels were within physiological ranges (standard references of $5-15 \mathrm{mg} / \mathrm{l}$ and $8-15 \mathrm{mg} / \mathrm{l}$ for vitamins $\mathrm{C}$ and $\mathrm{E}$, respectively, and $160-450 \mu \mathrm{mol} / 1$ for UA). SH levels ranged from 421 to $884 \mu \mathrm{mol} / 1$. Minimum and maximum urinary isoprostane values were 1.27 and $4.20 \mathrm{ng} / \mathrm{mg}$ creatinine (Table 1 ).

After HFM-PB ingestion, both UA (Fig. 1 (a)) and $\mathrm{SH}$ (Fig. 1(b)) levels were significantly increased, reaching maximum concentrations after $2(38 \% ; P<0.01)$ and $4 \mathrm{~h}(70 \%$; $P<0.001)$, respectively. After the consumption of the meal along with the two fruit juice drinks, UA production was significantly reduced with respect to placebo beverage consumption (Fig. 1(a)) $8 \mathrm{~h}$ after HFM-AB2 ingestion $(P<0.05)$. $\mathrm{SH}$ levels were also reduced with respect to the placebo beverage between 0.5 and $2 \mathrm{~h}(P<0.05$ at 0.5 and $1 \mathrm{~h}$; $P<0.01$ at $2 \mathrm{~h})$ by HFM-AB1 ingestion and between 2 and $8 \mathrm{~h}(P<0.05$ at 2,4 and $8 \mathrm{~h})$ by HFM-AB2 ingestion (Fig. 1 (b)).

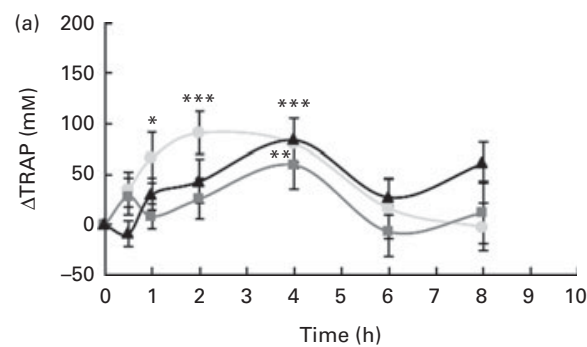

The HFM-PB intervention significantly increased plasma NEAC as measured by TRAP $4 \mathrm{~h}$ after meal ingestion (7\%; $P<0.01$ ) (Fig. 2(a)). After HFM-AB1 consumption, plasma TRAP was increased between $1(P<0.05)$ and $2 \mathrm{~h}$ (peak of increase, $8 \%, P<0.001)$ and started to decrease towards basal levels after $4 \mathrm{~h}(P<0 \cdot 01)$ (Fig. 2(a)). In the case of HFM-AB2, based on lower in vitro NEAC, no statistically significant changes in TRAP values were recorded (Fig. 2(a)). None of the test meals was found to significantly increase plasma NEAC as measured by FRAP (Fig. 2(b)). Plasma vitamin C, $\alpha$-tocopherol, cryptoxanthin, lycopene, and $\alpha$ - and $\beta$-carotene levels were not affected by the test meals (data not shown).

The urinary excretion of UA was significantly increased from baseline after HFM-PB and HFM-AB2 ingestion within $8 \mathrm{~h}(P<0.05$ in both cases) (Fig. 3(a)). However, UA excretion was not increased with respect to the placebo beverage by either of the antioxidant beverages (Fig. 3(a)). Among the three meals, only the HFM-AB1 intervention significantly increased the urinary excretion of antioxidants as indicated by raised urinary FRAP values measured within $8 \mathrm{~h}$ of ingestion (35\%,P<0.01) (Fig. 3(b)). None of the test meals had an effect on the urinary excretion of isoprostanes (Table 4).

\section{Discussion}

In a previous work, we had reported that the body responded through an inflammatory reaction to a HFM designed to contain no antioxidants, accompanied by an increased production of the endogenous antioxidants UA and SH, but not to the supplementation of the circulating antioxidants vitamin $\mathrm{C}$

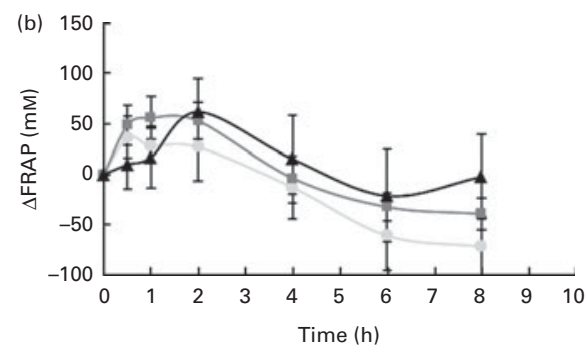

Fig. 2. Absolute changes $(\Delta=$ change $v$. T0) in plasma (a) total radical-trapping antioxidant parameter (TRAP) and (b) ferric reducing antioxidant power (FRAP) values following the consumption of the high-fat meal (HFM) + placebo beverage $(-\downarrow), \mathrm{HFM}+$ antioxidant beverage $1(-)$ ) and HFM + antioxidant beverage 2 $(--)$ in fourteen healthy, overweight volunteers. Values are means, with their standard errors represented by vertical bars. Mean value was significantly different from that before meal intake: * $P<0.05,{ }^{\star \star} P<0.01,{ }^{\star \star \star} P<0.001$. 
(a)

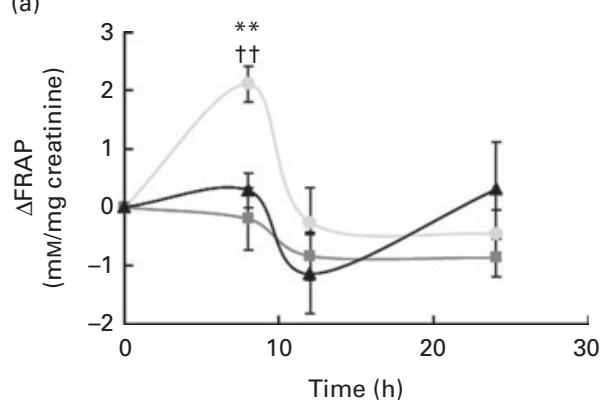

(b)

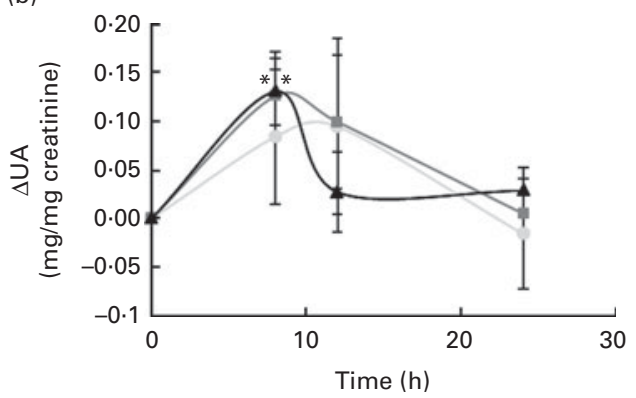

Fig. 3. Absolute changes ( $\Delta=$ change $v$. T0) in urinary (a) ferric reducing antioxidant power (FRAP) and (b) uric acid (UA) following the consumption of the highfat meal (HFM) + placebo beverage ( $\_$) , HFM + antioxidant beverage $1(--)$ and HFM + antioxidant beverage 2 ( - - ) in fourteen healthy, overweight volunteers. Values are means, with their standard errors represented by vertical bars. Mean value was significantly different from that before meal intake: ${ }^{\star} P<0 \cdot 05$, ${ }^{* \star} P<0.01$. †† Mean value for HFM + antioxidant beverage 1 was significantly different from that of the placebo beverage within a single time point $(P<0.01)$.

and $\mathrm{E}$ or carotenoids ${ }^{(6)}$. In the present study, we showed that the concomitant consumption of antioxidant-rich beverages with a meal may inhibit this response, possibly due to the preferential utilisation of ready-to-use antioxidant molecules deriving from foods.

We investigated the effects of two distinct beverages, differing in their in vitro antioxidant capacities and total phenolic contents, with AB1 having higher values, on the endogenous antioxidant response induced by HFM. In agreement with in vitro data, the present results suggest that $\mathrm{AB} 1$ also resulted in a higher antioxidant effect in vivo. Indeed, higher plasma TRAP levels and urinary FRAP values were recorded after AB1 ingestion than after AB2 ingestion. Moreover, AB2 led to the urinary excretion of UA, which was higher with respect to that observed for $\mathrm{AB} 1$ and similar to that recorded after the consumption of the placebo beverage. This indicates that the less antioxidant drink exerts a lower inhibitory effect on UA production with respect to the more antioxidant one. Although in vitro data cannot be readily applied to in vivo models, some evidence exists that high-antioxidant diets are able to better protect from diseases than low-antioxidant ones ${ }^{(25,26)}$. However, the bioavailability of antioxidant molecules from foods is normally complicated by a number of confounding factors, including metabolic fate after ingestion and food component interactions, making it often difficult to correlate higher in vitro antioxidant capacities with more incisive in vivo health effects. In general, both antioxidant beverages displayed remarkably higher antioxidant capacities when compared with commercially available fruit juices ${ }^{(27)}$.

In line with previous observations made by our group ${ }^{(6)}$, plasma UA and SH levels were significantly increased after HFM consumption. UA and protein $\mathrm{SH}$ groups play a major role in the maintenance of extracellular redox homeostasis, accounting for the majority of the endogenous plasma antioxidant capacity ${ }^{(11)}$. Although at physiological levels UA is an active component of the antioxidant network, altered plasma concentrations have been reported to be associated with the development of a number of inflammation-related diseases, including gout and renal and cardiovascular impairment in hyperuricaemic conditions as well as neurodegenerative diseases in hypouricaemic states ${ }^{(28)}$. Similarly, the redox state of $\mathrm{SH} /$ disulphide couples is finely regulated inside the human body, and alterations in this equilibrium have been reported to be associated with early atherosclerosis and renal failure (increased oxidised forms) or HIV (increased reduced forms $)^{(29-31)}$. Thus, while the activation of endogenous antioxidant mechanisms of defence in response to acute postprandial stress can be protective on consuming a single meal, the consistent alteration of the endogenous redox homeostasis induced by these responses may pave the way for negative effects in the long term. Data from the present study indicate that fruit drink beverages may prevent the development of non-physiological levels of UA and $\mathrm{SH}$ under postprandial stress conditions, thereby protecting from the risk of related diseases.

As has been mentioned above, our experimental meal was specifically designed to have a negligible purine content. Thus, the increase in UA levels observed after HFM-PB consumption was more likely to be the result of endogenously produced UA, rather than diet-induced UA. This hypothesis is further supported by the fact that we found a significantly increased UA concentration after HFM consumption, but not after the consumption of the HFM and fruit-based drinks. Similarly, Howatson et al. ${ }^{(32)}$ reported significant increases in plasma UA concentration in recreational marathon runners after a marathon and found that this increase was not observed in the runners if they consumed a tart cherry juice $5 \mathrm{~d}$ before, the day of and $48 \mathrm{~h}$ following the race. Although the mechanism has been poorly investigated so far, altogether these data seem to indicate that UA is endogenously produced under oxidative stress conditions, but when

Table 4. Urinary isoprostane concentrations ( $\mathrm{ng} / \mathrm{mg}$ creatinine) before and after the consumption of the high-fat meal (HFM) + placebo beverage (PB), HFM + antioxidant beverage 1 (AB1) and HFM + antioxidant beverage 2 (AB2)

(Mean values and standard deviations, $n$ 14)

Time after meal ingestion

\begin{tabular}{|c|c|c|c|c|c|c|c|c|}
\hline & \multicolumn{2}{|c|}{$\mathrm{Oh}$} & \multicolumn{2}{|c|}{$8 \mathrm{~h}$} & \multicolumn{2}{|c|}{$12 \mathrm{~h}$} & \multicolumn{2}{|c|}{$24 \mathrm{~h}$} \\
\hline & Mean & SD & Mean & SD & Mean & SD & Mean & SD \\
\hline HFM-PB & $2 \cdot 6$ & $1 \cdot 1$ & $3 \cdot 1$ & $1 \cdot 2$ & $2 \cdot 4$ & 0.9 & 3.30 & $2 \cdot 5$ \\
\hline HFM-AB1 & $2 \cdot 7$ & 0.9 & $3 \cdot 1$ & 0.9 & 2.9 & 0.8 & $2 \cdot 74$ & $1 \cdot 3$ \\
\hline HFM-AB2 & $2 \cdot 7$ & 1.0 & $3 \cdot 4$ & 1.4 & $3 \cdot 3$ & $2 \cdot 0$ & $2 \cdot 0$ & 1.4 \\
\hline
\end{tabular}


dietary antioxidants are available, UA production is inhibited, ensuring the maintenance of physiological circulating levels. Accordingly, we found that the fruit beverages did not increase urinary UA levels with respect to the placebo beverage, further suggesting that there was an inhibition of production and not an increased excretion. This hypothesis is also in line with recent animal studies that reported a hypouricaemic effect of dietary polyphenols due to the inhibition of the liver xanthine oxidoreductase, the key enzyme in the catabolism of purines to urate $e^{(33,34)}$

The present results indicate that the levels of circulating reduced thiol forms were increased $2-4 \mathrm{~h}$ after HFM ingestion. Similarly, Blanco et $a l .{ }^{(35)}$ recorded diurnal variations in plasma $\mathrm{SH}$ concentrations and showed that these variations were closely related to mealtimes, reporting peak values $3 \mathrm{~h}$ after meal consumption. Under oxidative stress conditions, protein thiol groups are prone to oxidation, which is normally prevented by the thiolation of proteins and subsequent release of reduced forms from tissues for the maintenance of redox balance $^{(36)}$. Thus, the increased $\mathrm{SH}$ levels observed after HFM ingestion may be related both to compensatory events and to the increase in circulating protein levels, including dietary protein levels. Nonetheless, the fact that we did not observe a significant increase in $\mathrm{SH}$ levels when the same HFM was consumed together with antioxidant beverages seems to indicate that the dietary antioxidants may have prevented the oxidation of protein thiol groups, reducing the need for endogenous compensatory events. Further investigations aimed at measuring the circulating levels of single plasma thiol species are needed in order to clarify the role of $\mathrm{SH}$ in the postprandial phase.

We found that the ingestion of HFM led to an increase in plasma NEAC after $4 \mathrm{~h}$. When the HFM was consumed along with the beverages, the pattern of the increase in NEAC changed, indicating earlier increments mainly between 1 and $2 \mathrm{~h}$. Although we cannot indicate which components of the fruit juice drinks were responsible for this effect from the present experiments, the lack of change in the vitamin pool after the consumption of the three meals allows us to postulate that other compounds present in the two beverages, including phenols and their metabolites, may have contributed to the increase in NEAC. Similarly, the consumption of phenol-rich foods and beverages has been reported to acutely increase plasma NEAC $0.5-2 \mathrm{~h}$ after ingestion ${ }^{(37)}$. These data indirectly suggest that a rapid uptake of exogenous molecules endowed with antioxidant activities may have occurred, thereby potentially protecting the body from postprandial stress through a variety of possible mechanisms. Recently, we have found that the ingestion of a mixed fruit juice drink delivering a high concentration of anthocyanins and flavonols significantly reduced the acute production of inflammatory cytokines induced by $\operatorname{HFM}^{(7)}$. Other authors have found that phenols from orange juice significantly reduced plasma endotoxin concentration, Toll-like receptor expression and reactive oxygen species generation by polymorphonuclear cells induced by a high-fat, high-carbohydrate meal ${ }^{(38)}$. Similarly, other studies have reported that dietary polyphenols may reverse the inflammatory effect of postprandial stress induced by fat overload and protect from increased cardiovascular risk ${ }^{(12,24)}$

It has been suggested that dietary fructose may be responsible for increased plasma UA levels, and this, but not dietary antioxidants, should explain the higher plasma NEAC after fruit consumption ${ }^{(39)}$. In the present study, we observed an increase in UA levels when volunteers consumed the HFM-PB, but not when the HFM was consumed with the fruit juice drinks. Therefore, the increase in NEAC observed in the present study following antioxidant beverage consumption cannot be attributed to plasma UA concentrations or to the induction of UA production by fructose, further confirming the idea that dietary antioxidants are probably responsible for this effect. Accordingly, Tulipani et al. ${ }^{(40)}$ showed that dietary consumption of strawberries raised plasma NEAC and not UA levels in healthy humans. Furthermore, Otaolaurruchi et al. ${ }^{(41)}$ recorded an increase in plasma FRAP after $7 \mathrm{~d}$ of red wine consumption, without any changes in UA concentration.

In conclusion, fruit-based juice drinks, providing an exogenous and ready-to-use source of redox molecules, prevent the endogenous antioxidant response to a single HFM, by inhibiting the production of $\mathrm{UA}$ and $\mathrm{SH}$.

To our knowledge, this is the first time that the effects of dietary antioxidants on the endogenous antioxidant response to HFM have been investigated. Our findings are of scientific and practical relevance, highlighting the potential value of consuming antioxidant-rich foods or beverages during mealtime in order to avoid non-physiological plasma UA and $\mathrm{SH}$ levels that may lead to chronic inflammation and CVD. Further research is needed to shed light on the mechanisms through which dietary antioxidants can blunt the endogenous oxidative stress induced by high-fat and -energy meals.

\section{Acknowledgements}

We thank all the volunteers for their participation in the present study. The present study was financially supported by The Coca-Cola Company, Atlanta, USA. The Coca-Cola Company had no role in the design, analysis or writing of this article.

The authors' contributions are as follows: C. M. wrote the paper and contributed to the statistical analysis; I. P. conducted the research, performed the statistical analysis and assisted with manuscript preparation; A. R., D. V. V., E. C., G. C. and E. T. conducted the research; M. S. designed the research (project conception, overall research plan development and study oversight) and assisted with manuscript preparation. All authors read and approved the final manuscript.

None of the authors has any conflicts of interest.

\section{References}

1. Burton-Freeman B (2010) Postprandial metabolic events and fruit-derived phenolics: a review of the science. Br J Nutr 104, S1-S14.

2. Node K \& Inoue I (2009) Postprandial hyperglycemia as an etiological factor in vascular failure. Cardiovasc Diabetol $\mathbf{8}$ 23-33. 
3. Burdge GC \& Calder PC (2005) Plasma cytokine response during the postprandial period: a potential causal process in vascular disease? BrJ Nutr 93, 3-9.

4. Eberly LE, Stamler J \& Neaton JD (2003) Relation of triacylglycerol levels, fasting and non-fasting, to fatal and non-fatal coronary heart disease. Arch Intern Med 163, 1077-1083.

5. Nappo F, Esposito K, Cioffi M, et al. (2002) Postprandial endothelial activation in healthy subjects and in type 2 diabetic patients: role of fat and carbohydrate meals. $J \mathrm{Am}$ Coll Cardiol 39, 1145-1150.

6. Miglio C, Peluso I, Raguzzini A, et al. (2013) Antioxidant and inflammatory response following high fat meal consumption in overweight subjects. EJN 52, 1107-1114.

7. Peluso I, Raguzzini A, Villano DV, et al. (2012) High fat meal increase of IL-17 is prevented by ingestion of fruit juice drink in healthy overweight subjects. Curr Pharm Des 18, 85-90.

8. Schinkovitz A, Dittrich P \& Wascher TC (2001) Effects of a high-fat meal on resistance vessel reactivity and on indicators of oxidative stress in healthy volunteers. Clin Physiol 21, 404-410.

9. Bae JH, Bassenge E, Kim KB, et al. (2001) Postprandial hypertriglyceridemia impairs endothelial function by enhanced oxidant stress. Atherosclerosis 155, 517-523.

10. Carr A \& Frei B (2000) The role of natural antioxidants in preserving the biological activity of endothelium-derived nitric oxide. Free Radic Biol Med 28, 1806-1814.

11. Bartosz G (2010) Non-enzymatic antioxidant capacity assays: limitations of use in biomedicine. Free Radic Res 44, 711-720.

12. Ellis CL, Edirisinghe I, Kappagoda T, et al. (2011) Attenuation of meal-induced inflammatory and thrombotic responses in overweight men and women after 6-week daily strawberry (Fragaria) intake. A randomized placebo-controlled trial. $J$ Atheroscler Thromb 18, 318-327.

13. Ghiselli A, Serafini M, Maiani G, et al. (1995) A fluorescencebased method for measuring total plasma antioxidant capability. Free Radic Biol Med 18, 29-36.

14. Benzie IFF \& Strain JJ (1996) The ferric reducing ability of plasma (FRAP) as a measure of antioxidant power: the FRAP assay. Anal Biochem 239, 70-76.

15. Ellman GL (1959) Tissue sulfhydryl groups. Arch Biochem Biophys 82, 70-77.

16. Margolis SA \& Schapira RM (1997) Liquid chromatographic measurement of L-ascorbic acid and D-ascorbic acid in biological samples. J Chromatogr B Biomed Sci Appl 690, 25-33.

17. Stacewicz-Sapuntzakis M, Bowen PE, Kikendall JW, et al. (1987) Simultaneous determination of serum retinol and various carotenoids: their distribution in middle-aged men and women. J Micronut Anal 3, 27-45.

18. Maiani G, Pappalardo G, Ferro-Luzzi A, et al. (1995) Accumulation of beta-carotene in normal colorectal mucosa and colonic neoplastic lesions in humans. Nutr Cancer 24, 23-31.

19. Mullen W, Borges G, Lean ME, et al. (2010) Identification of metabolites in human plasma and urine after consumption of a polyphenol-rich juice drink. J Agric Food Chem 58, 2586-2595.

20. Singleton VL \& Rossi JA (1965) Colorimetry of total phenolics with phosphomolybdic phosphotungstic reagents. Am J Enol Vitol 16, 144-158.

21. Italian Society of Human Nutrition (2012) Livelli di Assunzione Raccomandati di Energia e Nutrienti per la Popolazione Italiana (LARN) (Recommended Levels of Intake of Nutrients and Energy for the Italian Population). Summary presented at the XXXV National Congress, Bologna, Italy.

22. Choi HK, Liu S \& Curhan G (2005) Intake of purine-rich foods, protein, and dairy products and relationship to serum levels of uric acid: the Third National Health and Nutrition Examination Survey. Arthritis Rheum 52, 283-289.
23. German Research Institute for Food Chemistry (2005) SouciFachmann-Kraut-online database. http://www.sfk-online.net (accessed January 2012).

24. Ghanim H, Sia CL, Upadhyay M, et al. (2010) Orange juice neutralizes the proinflammatory effect of a high-fat, highcarbohydrate meal and prevents endotoxin increase and Toll-like receptor expression. Am J Clin Nutr 91, 940-949.

25. Serafini M, Jakszyn P, Luján-Barroso L, et al. (2012) Dietary total antioxidant capacity and gastric cancer risk in the European prospective investigation into cancer and nutrition study. Int J Cancer 131, E544-E554.

26. Del Rio D, Agnoli C, Pellegrini N, et al. (2011) Total antioxidant capacity of the diet is associated with lower risk of ischemic stroke in a large Italian cohort. J Nutr 141, 118-123.

27. Pellegrini N, Serafini M, Colombi B, et al. (2003) Total antioxidant capacity of plant foods, beverages and oils consumed in Italy assessed by three different in vitro assays. J Nutr 133, 2812-2819.

28. Kutzing MK \& Firestein BL (2008) Altered uric acid levels and disease states. J Pharmacol Exp Ther 324, 1-7.

29. Ashfaq S, Abramson JL, Jones DP, et al. (2006) The relationship between plasma levels of oxidized and reduced thiols and early atherosclerosis in healthy adults. $\mathrm{J} \mathrm{Am} \mathrm{Coll} \mathrm{Cardiol}$ 47, 1005-1011.

30. Hultberg B, Andersson A \& Arnadottir M (1995) Reduced, free and total fractions of homocysteine and other thiol compounds in plasma from patients with renal failure. Nephron 70, 62-67.

31. Müller F, Svardal AM, Aukrust P, et al. (1996) Elevated plasma concentration of reduced homocysteine in patients with human immunodeficiency virus infection. Am J Clin Nutr 63, 242-248.

32. Howatson G, McHugh MP, Hill JA, et al. (2010) Influence of tart cherry juice on indices of recovery following marathon running. Scand J Med Sci Sports 20, 843-852.

33. Mo SF, Zhou F, Lv YZ, et al. (2007) Hypouricemic action of selected flavonoids in mice: structure-activity relationships. Biol Pharm Bull 30, 1551-1556.

34. Haidari F, Mohammad Shahi M, Keshavarz SA, et al. (2009) Inhibitory effects of tart cherry (Prunus cerasus) juice on xanthine oxidoreductase activity and its hypouricemic and antioxidant effects on rats. Mal J Nutr 15, 53-64.

35. Blanco RA, Ziegler TR \& Carlson BA (2007) Diurnal variation in glutathione and cysteine redox states in human plasma. Am J Clin Nutr 86, 1016-1023.

36. Di Simplicio P, Frosali S, Priora R, et al. (2005) Biochemical and biological aspects of protein thiolation in cells and plasma. Antioxid Redox Signal 7, 951-963.

37. Serafini M, Miglio C, Peluso I, et al. (2011) Modulation of plasma non enzymatic antioxidant capacity (NEAC) by plant foods: the role of polyphenols. Curr Top Med Chem 11, 1821-1846.

38. Edirisinghe I, Banaszewski K, Cappozzo J, et al. (2011) Strawberry anthocyanin and its association with postprandial inflammation and insulin. BrJ Nutr 106, 913-922.

39. Lotito SB \& Frei B (2004) The increase in human plasma antioxidant capacity after apple consumption is due to the metabolic effect of fructose on urate, not apple-derived antioxidant flavonoids. Free Radic Biol Med 37, 251-258.

40. Tulipani S, Romandini S, Busco F, et al. (2009) Ascorbate, not urate, modulates the plasma antioxidant capacity after strawberry intake. Food Chem 117, 181-188.

41. Otaolaurruchi E, Fernández-Pachón MS, Gonzalez AG, et al. (2007) Repeated red wine consumption and changes on plasma antioxidant capacity and endogenous antioxidants (uric acid and protein thiol groups). J Agric Food Chem $\mathbf{5 5}$, 9713-9718. 